\title{
Suivi d'enfants exposés aux rayonnements ionisants dans le cadre de procédures radiologiques à visée diagnostique
}

\author{
H. BAYSSON ${ }^{1}$, N. JOURNY ${ }^{1}$, J.L. REHEL ${ }^{1}$, M. MEZZAROBBA ${ }^{1}$, \\ Y. BOUDJEMLINE ${ }^{2}$, D. BONNET ${ }^{2}$, A. BARUTEAU ${ }^{3}$, J. PETIT ${ }^{3}$, H.J. BRISSE ${ }^{4}$, \\ B. AUBERT ${ }^{1}$, D. LAURIER ${ }^{1}$, M.O. BERNIER ${ }^{1}$
}

(Manuscrit reçu le 17 avril 2012, accepté le 18 septembre 2012)

RÉSUMÉ Compte tenu de la radiosensibilité accrue des enfants aux rayonnements ionisants, notre objectif est d'évaluer les risques de cancers solides et de leucémies à long terme associés aux expositions médicales reçues pendant l'enfance. Deux études de cohorte sont réalisées par le Laboratoire d'épidémiologie de l'Institut de radioprotection et de sûreté nucléaire (IRSN). L'une, la « Cohorte Enfant Scanner » porte sur les enfants nés après le 01/01/1995 et exposés aux rayonnements ionisants lors de scanners réalisés entre 2000 et 2013 . L'autre porte sur les enfants exposés lors de procédures de cardiologie interventionnelle avant l'âge de 10 ans entre 2000 et 2013. Pour chacune des cohortes, une évaluation rétrospective individuelle de l'exposition sera réalisée par l'Unité d'expertise en radioprotection médicale de l'IRSN. Un suivi passif en termes de cancers solides et de leucémies sera réalisé grâce aux données des registres de cancers pédiatriques et aux données de mortalité. La « Cohorte Enfant Scanner » est constituée de plus 80000 enfants et s'inscrit dans le projet européen EPI-CT, qui regroupe 9 cohortes nationales. À terme, la cohorte des enfants en cardiologie interventionnelle regroupera plus de 8000 enfants. Ces études de cohortes, avec un suivi prolongé, permettront d'étudier l'impact sur la santé des expositions radiologiques reçues pendant l'enfance.

ABSTRACT Follow-up of children exposed to ionising radiation from medical procedures.

Given the increased radiosensitivity of children, our aim is to evaluate the risk of solid cancer and leukaemia associated with long-term medical exposure received during childhood. Two cohort studies were performed by the Laboratory of epidemiology, Institute of Radiation Protection and Nuclear Safety (IRSN). The first one, the "Cohort Child Scanner" includes all children born after 01/01/1995 and exposed to ionising radiation during medical scans performed between 2000 and 2013. The other one focuses on children exposed to ionising radiation during interventional cardiology procedures before the age of 10 years between 2000 and 2013 . For each cohort, a retrospective assessment of individual exposure will be conducted by the Medical Radiation Protection Expertise Unit of the IRSN. A passive follow-up in terms of solid cancer and leukaemia will be carried out using data from paediatric

\footnotetext{
Institut de radioprotection et de sûreté nucléaire (IRSN), BP 17, 92260 Fontenay-aux-Roses, France.

Centre de référence malformations cardiaques congénitales complexes, M3C, Hôpital Necker-Enfants Malades, 75015 Paris, France.

3 Centre de référence malformations cardiaques congénitales complexes, M3C, Centre Chirurgical Marie Lannelongue, 92350 Le Plessis-Robinson, France.

4 Institut Curie, Service de Radiodiagnostic, 26 rue d'Ulm, 75248 Paris, France.
} 


\begin{abstract}
cancer registries and from mortality data. The "Cohort Child Scanner" consists of over 80000 children and is part of the European project EPI-CT, which will gather data from 9 national cohorts. The cohort of children in interventional cardiology will include more than 8000 children. These cohort studies, with extended follow-up, will contribute to the assessment of the health impact of radiation exposure received during childhood.
\end{abstract}

Keywords: epidemiology / radiation / children / medical exposure / cancer

\title{
1. Introduction
}

Grâce aux progrès techniques réalisés dans le domaine de l'imagerie médicale, à l'introduction de nouvelles technologies (telles que les détecteurs numériques) et à la mise en œuvre des principes de radioprotection, les doses reçues lors des examens radiologiques ont pu être largement diminuées. Cependant l'exposition totale de la population n'est pas diminuée car on assiste aujourd'hui à la multiplication des actes médicaux, notamment aux âges extrêmes de la vie. En outre, des techniques diagnostiques irradiantes comme la scanographie ou la radiologie interventionnelle, délivrant des doses plus élevées que la radiologie conventionnelle sont de plus en plus utilisées, notamment chez les enfants. Compte tenu de leur radiosensibilité accrue par rapport à celle des adultes (UNSCEAR, 2006) et de leur espérance de vie plus grande, la conduite d'études épidémiologiques sur les effets à long terme des expositions médicales à visée diagnostique reçues pendant l'enfance, répond à une question importante de santé publique. Deux études de cohorte sont réalisées actuellement par l'Institut de radioprotection et de sûreté nucléaire (IRSN), au sein du Laboratoire d'épidémiologie (LEPID) en collaboration avec l'Unité d'expertise en radioprotection médicale (UEM). L'une, la « Cohorte Enfant Scanner », porte sur les enfants exposés aux rayonnements ionisants dans le cadre d'un ou plusieurs scanners. L'autre porte sur les enfants exposés aux rayonnements ionisants au cours d'une ou plusieurs procédures de cardiologie interventionnelle. Ces études permettront, d'une part, de quantifier le risque de cancers solides et de leucémies associé à des expositions à de faibles doses de rayonnements ionisants reçues pendant l'enfance et, d'autre part, de fournir des éléments nouveaux de radioprotection en pédiatrie.

\section{Contexte}

La principale source d'exposition de la population française aux rayonnements ionisants d'origine artificielle est liée aux expositions médicales, essentiellement à des fins diagnostiques (UNSCEAR, 2006). En France, la dose moyenne reçue par an et par habitant pour des examens diagnostiques est de l'ordre de 1,3 mSv, représentant $34 \%$ de l'exposition totale (Etard et al., 2010). Les tendances générales 
montrent un accroissement de l'exposition médicale de la population de l'ordre de 5 à $8 \%$ par an selon les pays (UNSCEAR, 2006). En 2007, une étude menée conjointement par l'IRSN et l'InVS (Institut de veille sanitaire), basée sur les données de l'assurance maladie et sur une enquête réalisée auprès d'un échantillon d'hôpitaux, a estimé qu'environ 74,6 millions d'actes diagnostiques utilisant les rayonnements ionisants ont été réalisés en France (tous âges confondus), soit 1,2 acte en moyenne par an et par habitant (Etard et al., 2010).

Selon Ait-Ali (Ait-Ali et al., 2010) les procédures les plus irradiantes sont les procédures de cardiologie interventionnelle à visée diagnostique et/ou thérapeutique et les scanners. La dose de radiation délivrée lors d'un examen scanner est en moyenne 10 à 100 fois plus élevée que celle délivrée lors d'un examen radiologique conventionnel. Les évolutions technologiques et les principes de radioprotection mis en œuvre ont certes permis de diminuer la dose délivrée au patient, mais la facilité et la rapidité d'acquisition des images encouragent la multiplication des examens, favorisant à terme l'accroissement des doses délivrées aux patients. Le scanner représente seulement $5 \%$ des examens réalisés par rayons $\mathrm{X}$, mais l'irradiation due au scanner représente selon les pays entre 40 et $67 \%$ de l'exposition médicale totale reçue (UNSCEAR, 2000). L'utilisation du scanner ne cesse de progresser, comme en atteste l'augmentation du nombre de scanographies réalisées aux États-Unis : de 18,3 millions en 1993 ; à 34,9 millions en 2000 et 62 millions en 2006 (IMV, 2006). Cette augmentation est aussi observée en pédiatrie, spécialité d'où émanent environ $10 \%$ de l'ensemble des examens scanner pratiqués annuellement aux États-Unis. En France, pour l'année 2007, environ 7,5 millions d'examen scanner ont été réalisés, dont près de 140000 en pédiatrie (Etard et al., 2010).

Une augmentation importante de la fréquence des procédures de cardiologie interventionnelle a également été observée ces dernières années (Le Tourneau et al., 2002 ; Van Aerschot et Boudjemline, 2012), car elles permettent, dans certains cas, de supplanter la chirurgie et donc de réduire considérablement les complications postopératoires, les coûts des hospitalisations tout en améliorant la survie des enfants atteints de cardiopathies congénitales. En Ile-de-France, 3 établissements (hôpital Necker, le centre chirurgical Marie Lannelongue et centre privé Jacques Cartier) réalisent actuellement des activités de cardiologie interventionnelle en pédiatrie. Environ 1600 cathétérismes sont réalisés par an dont 2/3 sont interventionnels (ARS, 2011). Ce chiffre de 1600 actes comprend néanmoins les éventuelles ré-interventions à l'âge adulte sur les cardiopathies congénitales. Si les techniques de cardiologie interventionnelle (Tab. I) ont permis des progrès incontestables dans le diagnostic et le traitement des cardiopathies chez les enfants, elles occasionnent une exposition aux rayonnements ionisants 
TABLEAU I

Indications des techniques de cathétérisme diagnostique et thérapeutique en pédiatrie (Van Aershot et Boudjemline, 2012).

Indications for Diagnostic and Therapeutic Cardiac Catheterization in paediatrics (Van Aershot and Boudjemline, 2012).

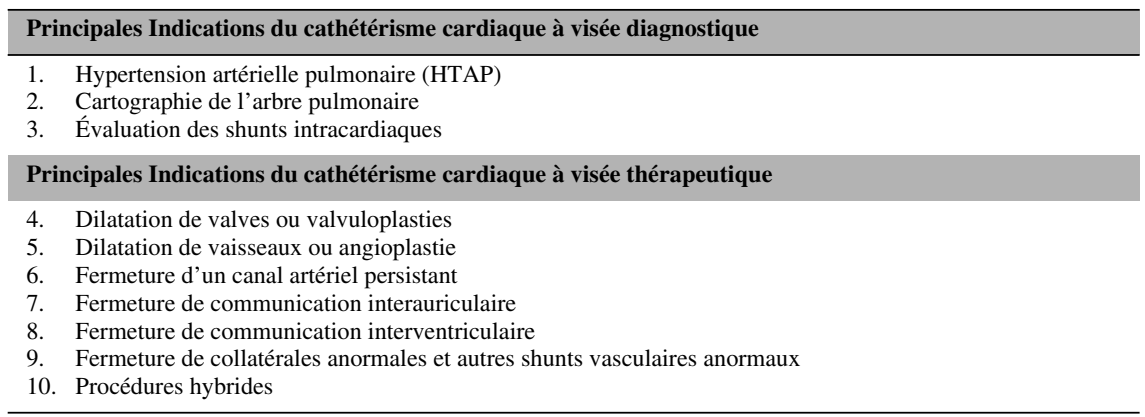

généralement supérieure à celle associée aux autres procédures utilisées en radiologie diagnostique (Onnasch et al., 2007).

Le tableau II présente les valeurs de dose efficace moyenne associées à des examens radiologiques et scanographiques réalisés de manière fréquente chez l'enfant et en cardiologie interventionnelle pédiatrique. Afin de disposer d'une information plus complète sur l'exposition des enfants lors d'actes diagnostiques, le tableau II présente également les doses délivrées aux organes lors de ces mêmes types d'examens réalisés chez un enfant de 5 ans. Les calculs ont été réalisés à l'aide de logiciels dédiés (CT Expo et PCXMC), en s'appuyant sur les recommandations dosimétriques en radiopédiatrie (Brisse et Aubert, 2009), à partir des guides de procédure des professionnels établis par la Société française de radiologie (SFR) pour les radiographies standards et à partir des protocoles d'acquisition d'images collectées dans le cadre de l'étude épidémiologique «Cohorte Enfant Scanner» (Bernier et al., 2012) pour les examens scanner.

Au cours des 30 dernières années, de nombreuses données épidémiologiques ont été rassemblées concernant des populations (tous âges confondus) exposées à des rayonnements ionisants d'origine médicale, à visée thérapeutique ou diagnostique (Schutz-Rath et al., 2008 ; Linet et al., 2009). Néanmoins ces études sont difficilement comparables entre elles puisqu'elles incluent des populations de tailles très différentes (de quelques centaines à quelques milliers de patients), hétérogènes en âge puisque certaines incluent des enfants mais également des jeunes adultes et de qualité méthodologique variable (Baysson et al., 2012). Les types d'irradiation considérés sont également très divers, que ce soit au niveau des organes cibles considérés ou au niveau de la période d'exposition, occasionnant 
TABLEAU II

Dose efficace moyenne par acte en radiologie conventionnelle, en scanographie et en cardiologie interventionnelle (Etard $\boldsymbol{e t}$ al., 2010 ; Ali-Aiti $\boldsymbol{e t}$ al. , 2010) et dose moyenne délivrée aux organes, pour un enfant de 5 ans (Etard $\boldsymbol{e t}$ al., 2010 ; Ali-Aiti $\boldsymbol{e t}$ al., 2010).

Mean effective dose values per examination, for conventional radiology, scanography and interventional cardiology (Etard $\boldsymbol{e t}$ al., 2010; Ali-Aiti $\boldsymbol{e t}$ al., 2010) and mean organ doses for a 5-year old child (Etard et al., 2010; Ali-Aiti et al., 2010).

\begin{tabular}{|c|c|c|}
\hline & $\begin{array}{l}\text { Dose efficace* moyenne } \\
(\mathrm{mSv})\end{array}$ & $\begin{array}{c}\text { Dose moyenne délivrée aux organes } \\
\text { (mGy) }\end{array}$ \\
\hline \multicolumn{3}{|c|}{ Radiologie conventionnelle } \\
\hline Radiographie du thorax* & 0,05 & $\begin{array}{l}\text { Poumons : } 0,07 \\
\text { Seins : } 0,02\end{array}$ \\
\hline Radiographie du pelvis & 1,2 & $\begin{array}{l}\text { Colon : } 0,2 \\
\text { Ovaires : } 0,15 \\
\text { Vessie : } 0,3\end{array}$ \\
\hline Radiographie de l'abdomen & 2 & $\begin{array}{l}\text { Intestin : } 0,4 \\
\text { Estomac : } 0,5 \\
\text { Vessie : } 0,5 \\
\text { Reins : } 0,1\end{array}$ \\
\hline $\begin{array}{l}\text { Radiographie panoramique } \\
\text { dentaire }\end{array}$ & 0,02 & \\
\hline Radiographie des membres & $<0,01$ & \\
\hline \multicolumn{3}{|l|}{ Scanographie } \\
\hline Scanner crânien & 1,1 & $\begin{array}{l}\text { Cerveau : } 20(\min 6-\max 47) \\
\text { Cristallins : } 30(\min 1-\max 60)\end{array}$ \\
\hline Scanner du thorax & 2,1 & Poumons, seins : $6(\min 1-\max 25)$ \\
\hline Scanner abdomino-pelvien & 8 & Ovaires : $12(\min 3-\max 30)$ \\
\hline \multicolumn{3}{|c|}{ Cardiologie Interventionnelle } \\
\hline Diagnostique & 4,6 & \\
\hline Thérapeutique & 6 & \\
\hline
\end{tabular}

des doses plus ou moins fortes. Les études de cohortes concernant des personnes soumises au cours de l'enfance à de nombreux examens radiologiques, dans le cadre d'un suivi de tuberculose ou de scoliose, sont les plus informatives et ont montré un risque de cancer du sein à l'âge adulte. Ce risque augmente avec la dose reçue et le nombre de radios (Boice et al., 1991 ; Howe et McLaughlin, 1995 ; Doody et al., 2000 ; Ronckers et al., 2010). Il est à noter que la dose cumulée reçue au niveau du sein était relativement importante, de 0,8 à 0,9 gray en moyenne dans le cadre du suivi de la tuberculose par radioscopie (Boice et al., 1991 ; Howe et McLaughlin, 1995) et de 0,11 gray pour le suivi de scoliose (Doody et al., 2000 ; Ronckers et al., 2010). Les études cas-témoins retrouvaient, quant à elles, des 
H. BAYSSON et al.

résultats divergents, certaines montrant une augmentation du risque de cancer après exposition aux examens radiologiques dans l'enfance (Shu et al., 1994 ; Infante-Rivard et Jacques, 2000 ; Bartley et al., 2010), d'autres ne retrouvant pas d'excès de risque (Meinert et al., 1999 ; Shu et al., 2002 ; Rajamaran et al., 2011).

Une première étude sur l'analyse du risque de développer un cancer après des examens répétés par scanographie, vient d'être publiée au Royaume-Uni (Pearce et al., 2012). Considérant les doses délivrées par les appareils de scanographie depuis 2001, les auteurs suggèrent que 2 à 3 examens par scanographie du crâne chez les enfants âgés de moins de 15 ans au moment de l'examen pourraient tripler leur risque de tumeurs cérébrales et que 5 à 10 examens par scanographie du crâne pourraient tripler leur risque de leucémies. Si les résultats obtenus pour les leucémies semblent cohérents avec ceux attendus par transposition des résultats issus de la cohorte des survivants d'Hiroshima et Nagasaki (Brenner et al., 2001 ; Brenner, 2010), ceux obtenus pour les tumeurs cérébrales sont plus de trois fois supérieures à ceux attendus. La possibilité d'un facteur confondant pour les tumeurs cérébrales ne peut être écartée. En particulier, il est à noter que la nature de la pathologie ayant nécessité un examen diagnostique par scanner, n'a pas pu être prise en compte dans cette étude britannique.

En ce qui concerne la cardiologie interventionnelle pédiatrique, le risque de cancer après cathétérisme cardiaque a été évalué dans deux études de cohorte. L'étude de Maclaughlin (Maclaughlin et al., 1993), portant sur 4891 enfants canadiens ayant bénéficié d'au moins un cathétérisme cardiaque avant l'âge de 18 ans entre les années 1946 et 1968, n'a pas mis en évidence d'excès de risque de cancer. L'étude de Modan (Modan et al., 2000), en revanche, fait état d'un excès de cancers et de lymphomes dans une cohorte de 674 enfants soumis à un cathétérisme cardiaque pour cardiopathie congénitale entre 1950 et 1970. Des limites méthodologiques (absence de dose estimée dans les deux études, suivi plus ou moins prolongé, types de procédure non individualisés) rendent toutefois l'interprétation de ces résultats divergents assez difficile.

\section{Méthodes}

Afin d'évaluer les risques à long terme de cancers solides et de leucémies après exposition aux rayonnements ionisants d'origine médicale pendant l'enfance, deux études de cohorte sont réalisées actuellement par le Laboratoire d'épidémiologie de l'IRSN. L'une, la « Cohorte Enfant Scanner », mise en place en collaboration avec la Société francophone d'imagerie pédiatrique et prénatale (SFIPP), porte sur les enfants exposés aux rayonnements ionisants dans le cadre d'un ou plusieurs scanners. L'autre porte sur les enfants exposés aux rayonnements ionisants au cours d'une ou plusieurs procédures de cardiologie interventionnelle. Dans les deux cas, 
la démarche est la même : il s'agit de suivre dans le temps une population définie (la cohorte) et d'enregistrer les pathologies survenant dans cette population, afin d'estimer les risques de cancer à long terme, risques associés à l'exposition aux rayonnements ionisants reçue dans le cadre de ces procédures radiologiques intervenues durant l'enfance.

\subsection{Constitution des cohortes à partir des données des services hospitaliers}

Pour la «Cohorte Enfant Scanner», les enfants sont recrutés à partir des plus grands centres de radiologie pédiatrique ou de radiologie adulte ayant une forte activité pédiatrique, répartis sur l'ensemble du territoire. Ainsi 21 centres hospitaliers universitaires (CHU), 20 en métropole et un dans les DOM-TOM (Ile de la Réunion) participent à l'étude. Les enfants inclus sont nés après le $1^{\mathrm{er}}$ janvier 1995 et ont été exposés à un premier scanner à partir de 2000, avant l'âge de 10 ans. L'inclusion se poursuit jusqu'en 2013. Les données recueillies pour chaque enfant sont les suivantes : nom, prénom, date de naissance, code postal de naissance, code postal de résidence, date de l'examen, zone anatomique exposée et service prescripteur. L'objectif est d'étudier le risque de cancer radio-induit chez des enfants indemnes de cancer; il nécessite un croisement des identifiants des enfants avec les registres de cancers pédiatriques: Registre national des tumeurs solides de l'enfant (RNTSE) et Registre national des hémopathies de l'enfant (RNHE), afin d'identifier et d'exclure de l'analyse les enfants qui auraient bénéficié d'un scanner pour le diagnostic ou le suivi d'un cancer. L'utilisation de données Programme médical de système d'information (PMSI) pour les enfants hospitalisés dans certains centres hospitaliers inclus a permis de vérifier l'exhaustivité des registres pour notre cohorte et de disposer d'informations cliniques complémentaires sur les pathologies présentées par les patients (Bernier, sous presse).

La cohorte des enfants ayant été exposés aux rayonnements ionisants au cours d'au moins une procédure de cardiologie interventionnelle est en cours de constitution auprès des services de cardiologie pédiatrique. Il concerne les enfants ayant bénéficié d'une procédure de cardiologie interventionnelle entre 2000 et 2013 et âgés de moins de 10 ans au moment de l'intervention de cardiologie. Les données collectées pour chaque enfant concernent son identification, les caractéristiques de l'intervention (date, motif, pathologie sous-jacente, type de procédure), les paramètres techniques tels que le temps de radioscopie et le produit dose surface (PDS) nécessaires à la reconstitution individuelle des doses reçues lors de l'intervention. 
H. BAYSSON et al.

\section{2. Évaluation rétrospective de l'exposition individuelle}

L'évaluation de l'exposition aux rayonnements ionisants est effectuée rétrospectivement pour chaque enfant inclus par l'unité d'expertise en radioprotection médicale (UEM) de l'IRSN.

Pour la «Cohorte Enfant Scanner», la reconstitution dosimétrique de l'exposition est réalisée à partir des protocoles d'acquisition d'image des services et en fonction du type de scanner utilisé. Cependant, pour la période la plus récente, avec la généralisation de systèmes de stockage performants de l'information dans les hôpitaux (PACS : Picture Archiving and Communication System), il est possible de disposer (informatiquement) des paramètres d'acquisition pour chaque examen réalisé, permettant ainsi de traiter l'information plus rapidement et sans erreur de saisie. Le calcul de la dose délivrée pour chaque examen permet ainsi de diminuer l'incertitude de la reconstitution dosimétrique. Plusieurs calculateurs de doses, validés par des mesures sur fantômes, permettent de calculer des doses de rayonnement délivrées aux organes à partir des paramètres d'acquisition de l'examen et du type de scanner utilisé. Le logiciel CT expo a été utilisé dans notre étude pour estimer les doses en tenant compte de l'âge de l'enfant (Stamm et Nagel, 2002).

En cardiologie pédiatrique, pour chaque enfant inclus dans la cohorte, une évaluation rétrospective individuelle des doses reçues dans le cadre des procédures de cardiologie interventionnelle sera réalisée en utilisant les paramètres techniques relevés in situ (temps de radioscopie et PDS). En cas de données manquantes, l'estimation de l'exposition reçue sera réalisée suivant les grands types de procédures de cardiologie interventionnelle préalablement définis et suivant les tranches de poids des enfants. Pour cela, des matrices «procédure-expositions » seront préalablement élaborées afin d'établir la correspondance entre les procédures de cardiologie interventionnelle fréquemment utilisées en pédiatrie et l'exposition radiologique potentiellement reçue en tenant compte du poids de l'enfant. En second lieu, les doses à l'organe, notamment pour le sein, le poumon, la thyroïde et l'œsophage seront calculées grâce au logiciel PCXMC (Tapiovaara et al., 1997).

\subsection{Suivi des enfants}

La limite principale des études de cohorte, en dehors du coût et du temps nécessaire à leur mise en œuvre, est le pourcentage de perdus de vue. Dans notre étude, le problème des perdus de vue est limité par le suivi passif de la cohorte. Le statut vital des enfants sera recueilli par croisement avec le Répertoire national d'identification des personnes physiques (RNIPP) afin de recueillir les statuts 
vitaux. Jusqu'à l'âge de 15 ans actuellement, mais jusqu'à 18 ans pour les années futures, la survenue de cancer ou de leucémie pourra être détectée grâce aux données des registres pédiatriques RNTSE et RNHE. Après 18 ans, compte tenu de l'absence de registre national du cancer chez l'adulte, le statut en termes de cancer ou de leucémie de la cohorte se fera d'une part sur l'analyse des causes de décès des personnes de la cohorte et d'autre part sur l'analyse des données de santé issues du Système national d'information inter-régimes de l'assurance maladie (SNIIR-AM).

Le suivi de la cohorte à long terme sera basé sur une mise à jour périodique du statut des enfants en termes d'incidence de cancers et de leucémies. Ce suivi doit être prolongé aussi longtemps que possible pour pouvoir étudier les pathologies tumorales susceptibles de se développer à long terme. Un décalage temporel (ou latence) entre l'exposition aux radiations et le développement de cancers de l'ordre de 5 à 10 ans sera prise en compte pour les cancers solides et de 2 à 5 ans pour les leucémies.

\subsection{Analyses statistiques}

L'étude de cohorte permet de suivre une population assez homogène (par exemple les enfants de moins de 10 ans ayant bénéficié d'une procédure de cardiologie interventionnelle) mais présentant une variabilité importante de l'exposition étudiée (puisque celle-ci varie notamment en fonction de la taille de l'enfant, la durée de chaque intervention, de l'équipement et de la technique employée). L'analyse fera appel aux méthodes d'analyse classiques des études de cohorte. Le rapport entre l'incidence des cancers solides et leucémies observée dans la cohorte et celle attendue dans la population générale française de même âge, sexe, et période de suivi, sera modélisé en utilisant les techniques de la régression Poissonnienne (Bouyer, 2010). Les données de référence (taux d'incidence dans la population générale) seront obtenues auprès des registres pédiatriques RNTSE et RNHE (Lacour et al., 2010). Les calculs seront stratifiés sur le sexe, la période calendaire de 5 ans et la classe d'âge. À terme, il s'agira de quantifier la relation dose-réponse entre l'exposition médicale reçue au cours de l'enfance et la survenue d'un cancer pendant l'enfance et/ou la mortalité par cancer à l'âge adulte. L'existence de facteurs modifiants éventuels (âge à l'exposition, sexe...) sera également étudiée. Une première analyse sera réalisée en 2012 pour la « Cohorte Enfant Scanner », soit jusqu'à 12 ans après la date d'inclusion, puis lors de points réguliers ultérieurs. Dès 2014, le suivi de la cohorte des enfants exposés aux rayonnements ionisants en cardiologie interventionnelle permettra d'apporter des premiers éléments de réponse sur l'incidence des cancers au sein de cette 
H. BAYSSON et al.

population. Des analyses ultérieures des données seront réalisées périodiquement (tous les 5 ans), au fur à mesure du vieillissement de la cohorte.

\subsection{Aspects éthiques}

Pour chacune des deux cohortes, un accord du Comité consultatif sur le traitement de l'information en matière de recherche dans le domaine de la santé (CCTIRS) et de la Commission nationale informatique et libertés (CNIL) a été obtenu. Pour la « Cohorte Enfant Scanner », l' autorisation initiale et son extension ont été obtenues, respectivement en décembre $2008\left(n^{\circ}\right.$ 908354) et en avril $2011\left(n^{\circ} 910431\right)$. Pour la cohorte des enfants exposés aux rayonnements ionisants dans le cadre de procédures de cardiologie pédiatrique, l'autorisation de la CNIL a été obtenue en décembre 2011 (autorisation $\mathrm{n}^{\circ}$ 911112).

\section{Résultats}

Pour la «Cohorte Enfant Scanner », plus de 80000 enfants ont d'ores et déjà été inclus avec un objectif total d'environ 100000 enfants. Une première description de la méthodologie et de la population inclue sur la période 2000-2006 dans 14 centres a été publiée (Bernier et al., 2012), détaillant les doses reçues selon le type de procédure. Les 27362 enfants inclus avaient entre 0 et 5 ans au premier examen ; $43 \%$ d'entre eux ont été exposés au scanner avant l'âge d'un an, dont $9 \%$ durant la période néonatale. Le nombre d'examen moyen par enfant était de 1,6 (Tab. III). Alors que $72 \%$ de la population n'avait fait l'objet que d'un seul examen, certains enfants ont été exposés à plusieurs examens, avec un maximum de 40 examens, pour un même enfant, sur la période considérée. L'examen le plus fréquemment réalisé était le scanner du crâne, qui représentait à lui seul $63 \%$ des procédures réalisées, le scanner du thorax a représenté $21 \%$ des procédures et le scanner abdominal ou pelvien $8 \%$ des procédures. Les autres procédures représentaient les $8 \%$ restants. Une grande variabilité des doses reçues a été observée selon les centres, montrant la nécessité d'homogénéiser les pratiques et d'optimiser leur mise en œuvre dans la mesure du possible.

En ce qui concerne la cohorte des enfants exposés aux rayonnements ionisants dans le cadre de procédures de cardiologie pédiatrique, le recrutement des enfants est actuellement en cours auprès des services de cardiologie pédiatrique ayant une activité de cardiologie interventionnelle. Le service de cardiologie pédiatrique de l'hôpital Necker (Paris) ainsi que celui du centre chirurgical Marie Lannelongue (Le Plessis-Robinson) qui traitent la plus grande partie des enfants du territoire national ont d'ores et déjà donné leur accord de participation à cette étude et la possibilité d'accéder aux données cliniques et, pour la période récente, aux 
TABLEAU III

Caractéristiques de la cohorte Enfant Scanner, France, 2000-2006 (Bernier et al., 2012).

Characteristics of participants in the "Cohort Enfant Scanner", France, 2000-2006

(Bernier et al., 2012).

\begin{tabular}{|c|c|c|}
\hline Caractéristiques & Enfants inclus $N=27362$ & Enfants éligibles $N=26189$ \\
\hline \multicolumn{3}{|c|}{ Nombre d'examens scanner par enfant } \\
\hline Moy (ET) & $1,6(1,7)$ & $1,5(1,4)$ \\
\hline 1 & $72 \%$ & $74 \%$ \\
\hline 2 & $16 \%$ & $15 \%$ \\
\hline 3 & $5 \%$ & $5 \%$ \\
\hline $3+$ & $7 \%$ & $6 \%$ \\
\hline \multicolumn{3}{|c|}{ Zone anatomique explorée } \\
\hline Tête & $63,0 \%$ & $65,7 \%$ \\
\hline Cou & $2,1 \%$ & $2,2 \%$ \\
\hline Thorax & $21,0 \%$ & $20,8 \%$ \\
\hline Abdomen et pelvis & $7,5 \%$ & $6,5 \%$ \\
\hline Extrémités & $0,2 \%$ & $0,2 \%$ \\
\hline Zones multiples & $3,7 \%$ & $2,3 \%$ \\
\hline Zone inconnue & $1,9 \%$ & $1,7 \%$ \\
\hline Autre & $0,6 \%$ & $0,6 \%$ \\
\hline
\end{tabular}

données dosimétriques des enfants concernés, a été validée. Le recrutement des enfants est actuellement en cours auprès de ces deux centres avant d'être étendu au niveau national. À terme, la cohorte sera constituée de plus de 8000 enfants. À ce jour, 4500 enfants ont été inclus dans la cohorte sur la période 2000-2011 à partir du service de cardiologie pédiatrique de l'hôpital Necker. En moyenne, chaque enfant a bénéficié de 1,3 procédure de cardiologie interventionnelle; ce qui représente un total de plus de 5000 procédures. Près de la moitié d'entre elles ont été réalisées pendant la première année de vie de l'enfant.

\section{Discussion}

Malgré la part importante prise par l'IRM, l'utilisation du scanner en pédiatrie conserve une place importante dans l'arsenal diagnostique puisqu'il permet d'obtenir des images en haute résolution, sur des champs d'exploration larges si besoin, et avec des temps d'acquisition très courts. Grâce aux nombreuses avancées technologiques, la cardiologie interventionnelle connait également un essor considérable. Dans certaines cardiopathies congénitales, elle permet de différer 
voire de supplanter la chirurgie, donc d'allier performance et thérapeutique moins invasive (Van Aerschot et Boudjemline, 2012). Parallèlement, certaines caractéristiques majeures propres à la pédiatrie sont à considérer. En effet, les enfants sont beaucoup plus sensibles aux radiations ionisantes que les adultes. L'étude des survivants des bombardements atomiques a montré que le risque de leucémie par unité de dose était de 5 à 10 fois plus élevé pour les irradiations subies avant l'âge de 5 ans, et que le surcroît de risque persistait à long terme, sur plusieurs dizaines d'années. Le délai de survenue des cancers solides serait également plus court lorsque l'irradiation a eu lieu pendant l'enfance que pour les expositions plus tardives (Preston et al., 2008). De même, une augmentation du risque de cancer de la thyroïde chez l'enfant après exposition à l'iode-131 a été clairement mise en évidence dans les suites de l'accident de Tchernobyl (Cardis et al., 2005). Les enfants ont également une espérance de vie longue, avec pour conséquence une probabilité plus élevée de développer un cancer. De plus, pour une procédure donnée, la dose reçue est plus élevée pour un enfant que pour un adulte, en raison de sa plus petite taille et d'organes plus proches les uns des autres que chez l'adulte (Linet et al., 2009). Enfin, on constate que l'ajustement des paramètres techniques d'acquisition à la morphologie du patient n'est pas systématiquement effectué lors de la réalisation des examens entrainant une surexposition des enfants (Linton et Mettler, 2003).

Ces caractéristiques justifient de s'intéresser prioritairement aux risques à long terme de cancers et de leucémies après exposition aux rayonnements ionisants d'origine médicale pendant l'enfance.

Des évaluations de risque (Brenner et al., 2001 ; Brenner, 2010) montrent également que les examens scanographiques réalisés durant l'enfance pourraient être associés à une augmentation du risque de cancer à long terme. Ainsi, pour des populations pédiatriques anglo-saxonnes ayant reçu un examen scanographique, Brenner et ses collègues ont estimé un excès de mortalité par cancer sur l'ensemble de la vie. Les risques de mortalité attribuables à un examen scanographique abdominal et cérébral chez un enfant de 1 an étaient respectivement de $0,18 \%$ et $0,07 \%$ (Brenner et al., 2001). En se basant sur les modèles de risque proposés dans la littérature (NRC, 2005), Ait-Ali (Ait-Ati et al., 2010) a estimé un risque attribuable vie entière de décès par cancer égal à 1 pour $1717(0,06 \%)$ chez les garçons recevant en moyenne $7,1 \mathrm{mSv}$ et égal à 1 pour $859(0,12 \%)$ chez les filles recevant en moyenne $9,4 \mathrm{mSv}$ entre 0 et 15 ans, au cours des procédures de cardiologie interventionnelle. Plus récemment, les résultats d'une étude épidémiologique britannique (Pearce et al., 2012) sur l'analyse du risque de développer un cancer après des examens répétés par scanographie pendant l'enfance montrent un excès de risque significatif de leucémies et de tumeurs cérébrales. 
S'agissant de risques attendus faibles, seules des études portant sur des effectifs importants pourront les mettre en évidence. Afin d'augmenter la puissance statistique des études prises isolément, le projet EPI-CT (Epidemiological Study to Quantify Risks for Paediatric Computerized Tomography and to Optimise Doses), actuellement en cours au niveau international, a pour objectif de rassembler les données de 9 cohortes nationales européennes d'enfants soumis à un ou plusieurs scanners, afin d'évaluer s'il existe un excès de cancer attribuable à cette pratique. C'est dans ce contexte que s'inscrit la «Cohorte Enfant Scanner» en France (Bernier et al., 2012). Des données similaires sont actuellement collectées en Grande-Bretagne (Pearce et al., 2012), en Allemagne (Krille et al., 2011) et dans d'autres pays européens. Au total, l'analyse statistique devrait porter sur plus d'un million d'enfants. In fine, les résultats obtenus pourront être mis en perspective avec d'autres études, de taille et de puissance statistique comparables. En cardiologie interventionnelle, il est prévu de rassembler les données originales collectées en France et au Royaume-Uni afin d'augmenter les effectifs et donc la robustesse des résultats statistiques. Actuellement, l'équipe de recherche du Dr M. Pearce (université de Newcastle, Grande-Bretagne) conduit une étude similaire auprès de patients âgés de moins de 22 ans et ayant bénéficié d'une procédure de cardiologie interventionnelle.

La mise en place des cohortes d'enfants exposés aux rayonnements ionisants dans le cadre de procédures diagnostiques ou thérapeutiques répond aux objectifs définis par le groupe d'experts High Level and Expert Group (HLEG) en ce qui concerne les questions actuelles en radioprotection (HLEG, 2009). En s'intéressant aux expositions reçues dans l'enfance, notre projet de recherche permettra d'apporter des éléments de réponse sur la variabilité individuelle du risque de cancer, et en particulier sur l'effet de l'âge à l'exposition. Dans le cadre des études menées sur les effets sur la santé des expositions médicales reçues pendant l'enfance, on peut également citer l'étude longitudinale française depuis l'enfance (ELFE) lancée en avril 2011. Le but de cette étude est de suivre 20000 enfants de la naissance à l'âge adulte afin d'analyser l'impact des différents facteurs familiaux, sociaux, scolaires, comportementaux, environnementaux, sanitaires ou nutritionnels sur leur développement physique, psychologique et social. Parmi ces facteurs, l'exposition médicale aux rayonnements ionisants sera collectée de façon prospective tout au long du suivi de l'enfant, grâce à un questionnaire précisant le type d'examen (radiographie, scanographie, IRM, etc.), le nombre et la localisation anatomique des examens. Cependant, le biais de mémoire pourra être important en fonction du délai entre la réalisation de l'examen et celle de l'entretien/questionnaire.

Dans la constitution de nos cohortes, la possibilité d'une reconstitution individuelle précise de l'exposition au scanner ou en cardiologie interventionnelle 
est un point très important afin de ne pas biaiser l'estimation du risque, si celui-ci est mis en évidence. Pour la « Cohorte Enfant Scanner », l'absence d'informations précises sur les protocoles radiologiques utilisés avant les années 2000 nous a conduits à commencer l'inclusion des enfants seulement à partir de 2000. Par contre, l'archivage d'information dosimétrique sur le PACS pour les années plus récentes nous permet d'envisager une reconstitution individuelle plus précise. Le développement de nouveaux logiciels de calcul tenant mieux compte de la morphologie des enfants dans le cadre du projet EPI-CT devrait encore améliorer la précision des doses estimées.

En cardiologie interventionnelle pédiatrique, il existe une grande variation de doses d'une procédure de cardiologie interventionnelle à l'autre, d'un enfant à l'autre (le poids de l'enfant au moment de l'intervention sera un élément déterminant à prendre en compte). La multiplication des procédures chez un même enfant peut entraîner à terme une dose cumulée relativement élevée. Il est également attendu une variation importante selon les services de cardiologie pédiatrique en fonction de l'utilisation de différentes machines et en fonction de l'expérience de l'opérateur. Ces différents aspects nous conduisent à évaluer rétrospectivement la dose reçue par chaque enfant au cours des procédures de cardiologie interventionnelle à partir des paramètres dosimétriques relevés in situ. Il est également prévu de croiser les données collectées en cardiologie interventionnelle avec celles de la «Cohorte Enfant Scanner » afin de considérer conjointement ces deux sources d'exposition.

La relation entre les expositions médicales diagnostiques aux rayonnements ionisants et le risque de cancer peut être masquée par la présence de facteurs de confusion qui sont associés à la fois à la maladie et à l'exposition (Linet $e t$ al., 2009). Ainsi, la pathologie sous-jacente présentée par l'enfant peut constituer un facteur de confusion. C'est par exemple le cas avec la trisomie-21 qui est associée au risque de leucémie (Tigay, 2009), mais également à une exposition aux rayonnements ionisants dans le cadre de bilan de malformations congénitales ou de pathologies spécifiques associées. C'est pourquoi il est essentiel de disposer d'informations cliniques sur les enfants inclus dans ces cohortes. Ces données peuvent être obtenues avec une bonne fiabilité via le système PMSI développé dans les hôpitaux (Bernier et al., sous presse). Le recours aux données de santé issues du SNIIR-AM paraît également intéressant pour le suivi de la cohorte à l'âge adulte mais nécessite des explorations complémentaires.

\section{Conclusion}

La question du risque de cancer associé à l'exposition médicale à visée diagnostique pendant l'enfance est particulièrement pertinente face à l'utilisation 
croissante des examens radiologiques, notamment des examens scanners, en pédiatrie. Les enfants représentent en effet une population particulièrement sensible aux rayonnements ionisants, caractérisée par une radiosensibilité de certains tissus accrue par rapport à celle des adultes, une espérance de vie longue, avec pour conséquence une probabilité plus élevée de développer un cancer. Des études de cohortes portant sur plusieurs milliers de patients exposés au scanner pendant l'enfance sont actuellement en cours dans le cadre du projet européen EPI-CT. Dans ce contexte, la «Cohorte Enfant Scanner » a été mise en place en France et, plus récemment, il a été décidé de constituer une cohorte d'enfants exposés aux rayonnements ionisants en cardiologie interventionnelle. Parallèlement, la poursuite des efforts pour réduire les doses et le nombre des examens radiologiques dans l'enfance reste essentielle, en vertu, en particulier, du principe ALARA (As Low As Reasonably Achievable), qui doit être mis en œuvre en imagerie pédiatrique.

\section{RÉFÉRENCES}

Ait-Ali L., Andreassi M., Foffa I. et al. (2010) Cumulative patient effective dose and acute radiationinduced chromosomal damage in children with congenital heart disease, Heart 96, 269-274.

ARS (2011) Agence régionale de santé Ile-de-France, Dossier de presse : Organisation de l'offre de soins en cardiologie interventionnelle.

Bartley K., Metayer C., Selvin S. et al. (2010) Diagnostic X-rays and risk of childhood leukemia, Int. J. Epidemiol. 39, 1628-1637.

Baysson H., Etard C., Brisse H., Bernier M.O. (2012) Expositions radiologiques à visée médicale diagnostique pendant l'enfance et risque de cancer : bilan des connaissances et perspectives, Archives de Pédiatrie 19, 64-73.

Bernier M.O., Rehel J.L., Brisse H., Wu-Zhou X., Caer-Lorho S., Jacob S., Chateil J.F., Aubert B., Laurier D. (2012) Radiation exposure from computed tomography in early childhood: a French large scale multicenter study, Brit. J. Radiol. 85, 53-60.

Bernier M.O., Mezzarobba M., Maupu E., Caër-Lorho S., Brisse H.J., Laurier D., Brunelle F., Chatellier G. (sous presse) Utilisation des données du programme de médicalisation des systèmes d'information (PMSI) dans les études épidémiologiques : application à la Cohorte Enfant Scanner, Rev. Epidemiol. Santé Publique.

Boice Jr J.D., Morin M.M., Glass A.G., Friedman G.D., Stovall M., Hoover R.N. et al. (1991) Diagnostic X-ray procedures and risk of leukemia, lymphoma, and multiple myeloma, JAMA 265, 1290-1294.

Bouyer J. (2010) Méthodes statistiques : Médecine - Biologie. Édition Estem, 351 p.

Brenner D., Elliston C., Hall E. et al. (2001) Estimated risks of radiation-induced fatal cancer from pediatric CT, Am. J. Roentgenol. 176, 289-296.

Brenner D.J. (2010) Slowing the increase in the population dose resulting from CT scans, Radiat. Res. $174,809-815$.

Brisse H.J., Aubert B. (2009) Niveaux d'exposition en tomodensitométrie multicoupes pédiatrique : résultats de l'enquête dosimétrique SFIPP/IRSN 2007-2088, J. Radiol. 90, 207-217.

Cardis E., Kesminiene A., Ivanov V. et al. (2005) Risk of thyroid cancer exposure to ${ }^{131}$ I in childhood, J. Natl. Cancer Inst. 97, 724-732. 
Doody M., Lonstein J.E., Stovall M. et al. (2000) Breast cancer mortality following diagnostic X-rays: findings from the US Scoliosis cohort study, Spine 25, 2052-2063.

Etard C., Sinno-Tellier S., Aubert B. (2010) Exposition de la population française aux rayonnements ionisants liée aux actes de diagnostic médical en 2007, Rapport InVS/IRSN.

HLEG (2009) High Level and Expert Group, Report of High Level and Expert Group, January 2009, http://www.hleg.de/fr.pdf.

Howe G.R., McLaughlin J. (1996) Breast cancer mortality between 1950 and 1987 after exposure to fractionated moderate-dose-rate ionizing radiation in the Canadian fluoroscopy cohort study and a comparison with breast cancer mortality in the atomic bomb survivors study, Radiat. Res. 145, 694-707.

ICRP Publication 60 (1991) 1990 Recommendations of the International Commission on Radiological Protection, Ann. ICRP 21 (1-3).

IMV (2006) Medical Information Division, Benchmark Report, CT 2006, IMV medical Information Division, Des Plaines, Illinois.

Infante-Rivard C., Jacques L. (2000) Empirical study of parental recall bias, Am. J. Epidemiol. 152, 480-486.

Krille L., Jahnen A., Mildenberger P. et al. (2011) Computed tomography in children: multicenter cohort study, Eur. J. Epidem. 26, 249-250.

Lacour B., Guyot-Goubin A., Guissou S., Bellec S., Désandes E., Clavel Incidence of childhood cancer in France: National Children Cancer Registries, 2000-2004, Eur. J. Cancer Prev. 19 (3), 173181.

Le Tourneau T., Blanchard D., Lablanche J.M., Monassier J.P., Morice M.C., Cribier A. et al. (2002) Evolution of interventional cardiology in France during the last decade (1991-2000), Arch. Mal. Coeur. Vaiss. 95, 1188-1194.

Linet M., Kim K., Rajamaran P. (2009) Children's exposure to diagnostic medical radiation and cancer risk: epidemiologic and dosimetric considerations, Pediatr. Radiol. 39 (Suppl 1), S4-S26.

Linton O., Mettler F. (2003) National Conference on dose reduction in CT, with an emphasis on pediatric patients, Am. J. Roentgenol. 181, 321-329.

McLaughlin J., Kreiger N., Sloan M., Benson L., Hilditch S., Clarke E. (1993) An historical cohort study of cardiac catheterization during childhood and the risk of cancer, Int. J. Epidemiol. 22, 584-591.

Meinert R., Kaletsch U. et al. (1999) Associations between childhood cancer and ionizing radiation: results of a population-based case-control study in Germany, Cancer Epidemiol. Biomarkers Prev. 8, 793-799.

Modan B., Keinan L., Blumstein T., Sadetzki S. (2000) Cancer following cardiac catheterization in childhood, Int. J. Epidemiol. 29, 424-428.

NRC (2005) National Research Council and Committee to Assess Health Risks from Exposure to Low Levels of Ionizing Radiation, Health Risks from Exposure to Low Levels of Ionizing Radiation: BEIR VII Phase 2 (Washington, DC: National Academy of Sciences).

Onnasch D., Schröder F., Fischer G., Kramer H. (2007) Diagnostic reference levels and effective dose in paediatric cardiac catheterization, $\mathrm{Br}$. J. Radiol. 80, 177-185.

Pearce M.S., Salotti J.A., Little M.P., McHugh K., Lee C., Kim K.P., Howe N.L., Ronckers C.M., Rajaraman P., Craft A.W., Parker L., Berrington de González A. (2012) Radiation exposure from CT scans in childhood and subsequent risk > of leukaemia and brain > tumours: a retrospective cohort study, Lancet.

Preston D.L., Cullings H., Suyana A. et al. (2008) Solid cancer incidence in atomic bomb survivors exposed in utero or as young children, J. Natl. Cancer Inst. 100, 428-436. 
Rajaraman P., Simpson J., Neta G. et al. (2011) Early life to diagnostic radiation and ultrasound scans and risk of childhood cancer: a case-control study, BMJ 342, d472.

Ronckers C., Land C., Miller J. et al. (2010) Cancer mortality among women frequently exposed to radiographic examinations for spinal disoders, Radiat. Res. 174, 83-90.

Schutz-Rath R., Hammer G.P., Blettner M. (2008) Are Pre- or postnatal diagnostic X-rays a risk factor for childhood cancer? A systematic review, Radiat. Environ. Biophys. 47, 301-312.

Shu X.O., Jin F., Linet M.S., Zheng W., Clemens J., Mills J., Gaol Y.T. et al. (1994) Diagnostic X-ray and ultrasound exposure and risk of childhood cancer, Br. J. Cancer 70, 531-536.

Shu X.O., Potter J.D. et al. (2002) Diagnostic X-rays and ultrasound exposure and risk of childhood acute lymphoblastic leukemia by immunophenotype, Cancer Epidemiol. Biomarkers Prev. 11, 177-185.

Stamm G., Nagel H.D. (2002) CT-expo--a novel program for dose evaluation in CT, Rofo 174, 15701576.

Tapiovaara M., Lakkisto M., Seromaa A. (1997) PCXMC, a PC-based Monte Carlo program for calculating patient doses in medical X-ray examinations, Stuk-A139.

Tigay J.H. (2009) A comparison of acute lymphoblastic leukemia in Down syndrome and non-Down syndrome children: the role of trisomy 21, J. Pediatr. Oncol. Nurs. 26, 362-368.

UNSCEAR (2000) United Nations Scientific Committee on the Effects of Atomic Radiation, Sources and effects of ionizing radiation, Volume I: Sources, New York, 2000.

UNSCEAR (2006) Effects of ionizing radiation, Report to the General Assembly of the United Nations, New York.

Van Aershot I., Boudjemline Y. (2012) Cathétérisme interventionnel et chirurgie cardiaque chez les enfants, Arch. Pédiatr. 19, 96-102. 